\title{
Optimal Power Flow with Considering Voltage Stability using Chaotic Firefly Algorithm
}

\author{
Yun Tonce Kusuma Priyanto ${ }^{1,2^{*}}$, Vicky Mudeng ${ }^{1}$, Muhammad Robith ${ }^{1}$ \\ ${ }^{1}$ Electrical Engineering Department, Kalimantan Institute of Technology, Balikpapan, Indonesia \\ ${ }^{2}$ National Center for Sustainable Transportation Technology, Indonesia \\ *Email:yuntonce@itk.ac.id
}

\begin{abstract}
In transportation technology, the development of electric vehicle is growing rapidly. In the future, the availability of electrical power is crucial because every electric tool needs electrical power. Power plant must provide electrical power for all consumer include an electric vehicle. Sustainability of electrical power transmission and distribution must be considered as critical due to its high power consumption in the community. One of the problem to supply electrical power is how to keep the system's voltage stability. Several variations on the load pattern and topological can lead to a substantial poor impact on the system. However, generation cost must be considered by utilities' operator. This paper demonstrates a recently developed metaheuristic method called Chaotic Firefly Algorithm (CFA). Our simulation shows that this method may perform better than several well-known metaheuristic methods. Therefore, CFA may become a promising method to solve optimal power flow considering voltage stability cases.
\end{abstract}

\section{Keywords}

Electric vehicle; Optimal power flow; Voltage stability; Artificial intelligent; L-index; Chaotic firefly algorithm

\section{Introduction}

The electric vehicle is growing rapidly in modern transportation technology. This condition makes the availability of electric vehicle charging very important. Therefore, the availability of electric power is the most important that we urge to produce and manage. This is why the sustainability of this power transmission and distribution is critical. In a regulated power system, utility's operators had to control the system in order to meet this demand. There are various objectives that must be achieved. Some of them are to minimize the power plant's operational cost [1,2], power losses [3] and to maximize the system's distance to voltage collapse [2]. There are also various constraints to be considered. Some of them are the generator, line, and voltage limits [1]. This problem is categorized as an optimization problem called Optimal Power Flow (OPF) problem in many works of literature [1-3].

One of some objectives considered in the OPF problem is the system's distance to voltage collapse [2]. Voltage collapse usually occurs after voltage instability. Voltage instability itself is a condition when the system is unable to maintain its steady acceptable voltages during normal operation and after being subjected to a disturbance [4]. Adverse impact from voltage collapse is low voltage profile in several parts of the system [4], in other words, decreased power quality. One of the well-recorded case studies on voltage collapse's impact is given in [5]. It is explained that some busses' voltage drops from 500 $\mathrm{kV}$ to $370 \mathrm{kV}$ in only 20 minutes, caused a massive 8 GW loss of load to recover the system.

There are several methods to solve the OPF problem. Generally, they are categorized as traditional and metaheuristic methods [6]. Each type of methods had several unique characteristics. One of them is their applicability when the problem is injected with some additional considerations. Traditional methods are unable to apply to those problems because these methods are derived with fixed assumptions and problem definition. Additional considerations that not defined nor assumed before must be modeled mathematically before traditional methods may start. This condition may turn into a disadvantage when it's too hard to construct their mathematical models, or their models are too complicated. In other hand, metaheuristic methods have several fixed mechanisms that not depended by the problem. It means that the problems did not affect how these methods work [7]. In some cases, when there are many complicated mathematical models encountered at the problem, this characteristic may become a huge advantage.

Since the Genetic Algorithm (GA) is a metaheuristic method, developed in 1960-1970 [8], there are many algorithms had been emerged. Some of them also applied to solve OPF problems [9-11], and even there is a review on metaheuristic methods used in various 
cases of OPF problem [6]. In [9], Abido demonstrated Particle Swarm Optimization (PSO) to solve the OPF problem with various objectives from reducing costs to voltage profile improvement.

In [10], Yang et al. demonstrated his famous method Firefly Algorithm (FA) to solve the economic dispatch problem, one of the subproblems of OPF. Reference [10] even gave some examples with mixed-variable cases which not related to economic dispatch. In [11], Vanitha et al. presented a hybrid Differential Evolution (DE) with Weighted Additive Fuzzy Goal Programming (WAFGP) to determine the best placement for STATCOM. The method is used to optimize the system's loadability when minimizing its power losses and STATCOM's installation cost. These three researches show the flexibility of metaheuristic methods able to be applied in various form of problems.

In this paper, we introduce a recently developed metaheuristic algorithm called Chaotic FA (CFA) for solving the OPF problem with considering voltage stability. This algorithm is an improved version of FA. To compare its performance to solve our problem, we also used FA and Accelerated PSO (APSO) to solve a similar problem. Simple statistical analyses will be performed as a comparison method to determine the best algorithm from those.

\section{OPF Problem}

OPF problem goal is to find the optimal settings for a given power system network to optimize some objectives such as total generator cost, system loss, bus voltage deviation, emission of generating units, number of control actions, and load shedding. On the other hand, the setting also satisfies the power flow equations, system security, and equipment operating limits [3]. There are several control variables that may be utilized to achieve this goal. Some of them are generators' real and reactive power output and voltage, transformer tap changing settings, phase shifters, switched capacitors, and reactors [3]. OPF problem of a power system has various formulations depending on considered objectives, constraints, and control variables. In this section, we define the formulation for this paper.

There are two objectives employed in this paper. They are total generation cost and power losses. In this paper, the total generation cost function is given in (1).

$f_{c}\left(P_{G}\right)=\sum_{k=1}^{N G} a P_{G k}^{2}+b P_{G k}+c$ where $f_{c}\left(P_{G}\right)$ is total generation cost function, $N G$ is the number of generating thermal units, $P_{G k}$ denotes real power output of $k$-th generating thermal units, and $a, b$, and $c$ are input-output characteristic coefficients [3]. Power losses function considered in this paper is given in (2).

$f_{1}\left(P_{G}\right)=\sum_{j=1}^{N D} P_{L j}-\sum_{k=1}^{N G} P_{G k}$

where $f_{l}\left(P_{G}\right)$ denotes power losses function, $N D$ is number of load bus, and $P_{L j}$ is real power demand at $j$ th bus.

In most works of literature discuss OPF problems, there are 4 four main constraints which have to be fulfilled: power flow equations, generator limits, line flow limits, and voltage limits [1-3]. Also, we define these constraints used in this paper. Power flow equations are defined in (3) and (4) for real and reactive power, respectively.

$\left(P_{G i}-P_{L i}\right)-\operatorname{Re}\left\{V_{i}\left(\sum_{k=1}^{N B} Y_{i k} V_{k}\right)^{*}\right\}=0$
$\left(Q_{G i}-Q_{L i}\right)-\operatorname{Im}\left\{V_{i}\left(\sum_{k=1}^{N B} Y_{i k} V_{k}\right)^{*}\right\}=0$

where $V_{i}$ is voltage at $i$-th bus in per-unit (p.u.) unit, $Y_{i k}$ denotes an element on $i$-th row and $k$-th column of admitance matrix in p.u. unit, and $N B$ is number of busses. $\operatorname{Re}\{x\}$ and $\operatorname{Im}\{x\}$ are operators to determine real and imaginary value of complex number $x[1]$.

Generator and voltage limits are given in (5) and (6), respectively. Generator limits applied to all thermal units that become control variable and voltage limits applied to all busses. max and min superscript means the maximum and minimum value of the mentioned variable, respectively [1].

$$
\begin{aligned}
& P_{G}^{\text {min }} \leq P_{G} \leq P_{G}^{\max } \\
& V_{i}^{\text {min }} \leq V_{i} \leq V_{i}^{\text {max }}
\end{aligned}
$$

\section{L-Index}

There are several methods to estimate the power system's voltage stability. One of them is classical Lindex. L-index is an indicator developed by Kessel and Glavitsch in 1986 to estimate power system's voltage stability [12]. According to the definition of voltage stability indices in [13], this indicator is categorized as 
a system variable based indices. It caused by usage of the information on a system such as bus voltage and power flow on the line. Therefore, L-index is capable of discovering weak busses in low computing time according to system variable based indices characteristic given by [13]. In this paper, we are using this index as a constraint for our problem.

L-index is defined for all load busses using (7) [12]

$L_{j b}=a b s\left\{1-\frac{\sum_{k=1}^{N G} F_{j k} V_{k g}}{V_{j b}}\right\}$

where $L_{j b}$ is L-index value for $j$-th load bus, $V_{k g}$ is voltage value on $k$-th non-load bus, and $V_{j b}$ is voltage value on $j$-th load bus. $a b s\{x\}$ is an operator to determine absolute value from complex number $x . F$ is a matrix defined in (8), where $F_{j k}$ is an element from $F$ matrix on $j$-th row and $k$-th column [12].

$F=-Y_{L L}^{-1} Y_{L G}$

In (9), $Y_{L L}$ is a part of the admittance matrix that defines admittance value between two load busses. Meanwhile, $Y_{L G}$ is a part of the admittance matrix that defines admittance value between load busses and nonload busses.

A power system estimated as unstable if maximum Lindex value achieved is more than one [12]. However, in this paper, we consider another parameter $L_{\max }$ where $0 \leq L_{\max }<1$. This parameter ensures the weakest bus is not too weak when the disturbance occurred; thus, it may reduce the variation of voltage instability. Finally, our L-index limit is given in (9):

$\max \left(L_{j b}\right) \leq L_{\max }$

\section{CFA}

CFA is the most recent developed metaheuristic method. This algorithm was designed by Gandomi et al. in 2013 for solving optimization problems [14]. This algorithm essentially runs like FA; however, it replaces constant parameters on FA with varying parameters at each iteration using chaotic sequences [14].

There are two main mechanisms in CFA. The first one is the FA's movement. For an $i$-th firefly or solution candidate, compare with another $j$-th firefly. If $i$-th fireflies' fitness value is worse than $j$-th fireflies' fitness value, then move it using (10) and define its new fitness value $[8,14]$. $x_{i}=x_{i}+\beta_{0} e^{-\gamma r^{2}}\left(x_{j}-x_{i}\right)+\alpha \varepsilon$

where $x_{i}$ is $i$-th firefly position vector, $\beta_{0}$ denotes attractiveness parameter, $\gamma$ is light absorption coefficient, $r$ is the distance between two fireflies, $\alpha$ is randomization parameter, and $\varepsilon$ denotes random numbers drawn from Gaussian distribution [14]. It is worth pointing out that distance $r$ defined here is not limited to Euclidean distance [15].

The other mechanism of CFA is applying a chaotic sequence to vary some parameters. In this paper, we vary $\beta_{0}$ value at the end of iteration using Gauss Map as suggested by [14]. Chaotic gauss map is given in (11), where $k_{n}$ is a number generated at $n$-th iteration and operator $[k]$ is floor function [14].

$k_{n+1}=\left\{\begin{array}{cc}0 & k_{n}=0 \\ \frac{1}{k_{n}}-\left[\frac{1}{k_{n}}\right] & k_{n} \neq 0\end{array}\right.$

Pseudocode of this algorithm is given in Figure 1.

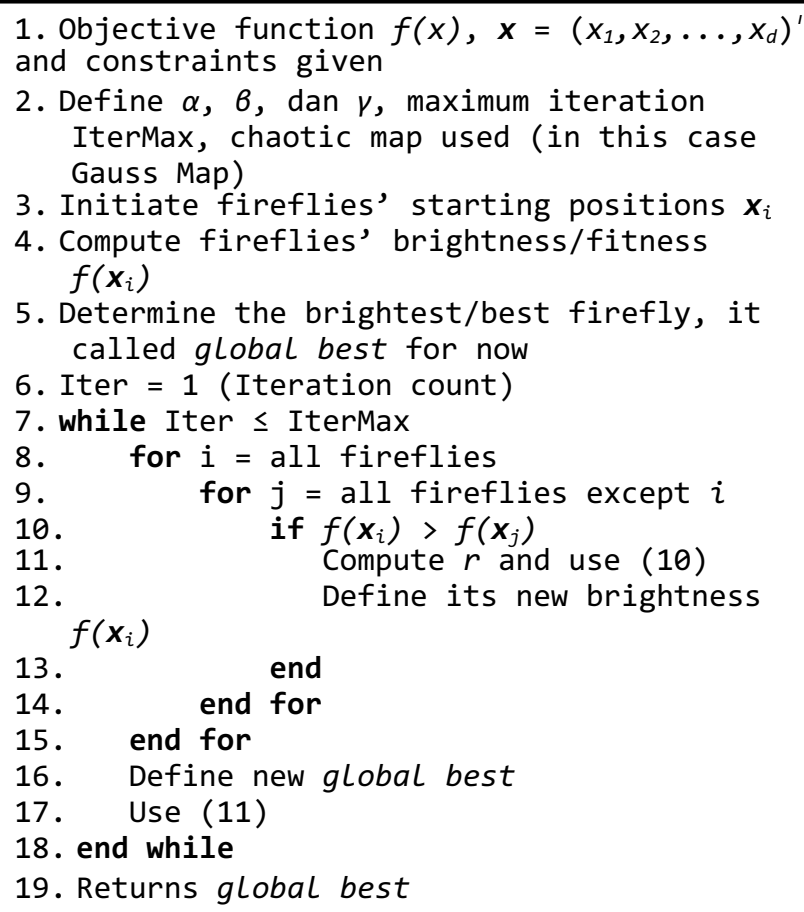

Figure 1 CFA's pseudocode

\section{$5 \quad$ Results and Discussion}

Before we show our simulation result, let us describe the system used in this paper. Here, we use $150 \mathrm{kV}$ Mahakam transmission power system installed at East Kalimantan [16]. However, load pattern case study considered here is a bit different. Load pattern case study considered here is the same as [16], an additional 50 MVAR reactive power on bus 1 . This addition will increase voltage instability variation to be occurred, 
according to [4]. Furthermore, to counter this additional reactive power, we simulate a capacitor bank with 50 MVAR maximum capacity installed in bus 1 . In this paper, there are four values of control variables that need to search: Real power generation $\left(P_{G}\right)$ at bus 3,5 , and 12 also capacitor capacity $\left(Q_{c a p}\right)$ at bus 1 . Here we also set $L_{\max }=0.2$.
Table 1 to 3 are given to clarify all needed data for our simulation, where Table 1 and Table 2 are exactly same with [16]. As shown in Table 2, each variable has different search ranges. This may cause slow movement in some variables with large search range when $\gamma$ is not well-defined. In order to counter it, we map our solution into [-1,1] so $\gamma$ may give uniform effect to all control variables.

Table 1 Line impedance data for each line

\begin{tabular}{|c|c|c|c|c|}
\hline \multicolumn{2}{|c|}{ Bus Number } & \multirow{2}{*}{ Resistance (p.u.) } & \multirow{2}{*}{ Impedance (p.u.) } & \multirow{2}{*}{ Susceptance (p.u.) } \\
\hline From & To & & & \\
\hline 1 & 2 & 0.058 & 0.167 & 0.002 \\
\hline 2 & 3 & 0.016 & 0.048 & 0.0008 \\
\hline 3 & 4 & 0.016 & 0.048 & 0.0008 \\
\hline 4 & 5 & 0.185 & 0.549 & 0.009 \\
\hline 5 & 6 & 0.020 & 0.060 & 0.001 \\
\hline 5 & 9 & 0.032 & 0.094 & 0.001 \\
\hline 6 & 7 & 0.038 & 0.115 & 0.002 \\
\hline 7 & 8 & 0.056 & 0.162 & 0.002 \\
\hline 9 & 10 & 0.017 & 0.052 & 0.0008 \\
\hline 10 & 11 & 0.111 & 0.328 & 0.005 \\
\hline 10 & 12 & 0.221 & 0.656 & 0.011 \\
\hline
\end{tabular}

Table 2 Generator characteristics and cost function

\begin{tabular}{cccccc}
\hline \multirow{2}{*}{ Bus } & $\boldsymbol{A}$ & $\boldsymbol{B}$ & $\boldsymbol{c}$ & \multicolumn{2}{c}{ Real Power Generation } \\
\cline { 4 - 6 } & & & & Min. $(\boldsymbol{M W})$ & Max. $\boldsymbol{M W})$ \\
\hline 3 & -16873 & 2288.5 & -15245 & 41 & 100 \\
7 & 0 & 1658.7 & 0 & 20 & 80 \\
12 & 0 & 2213.2 & 0 & 11 & 190 \\
\hline
\end{tabular}

Table 3 Load data for each bus

\begin{tabular}{ccc}
\hline \multirow{2}{*}{ Bus } & \multicolumn{2}{c}{ Load } \\
\cline { 2 - 3 } & Real Power $(\boldsymbol{M W})$ & Reactive Power $(\boldsymbol{M V A R})$ \\
\hline 1 & 53.994 & 68.396 \\
2 & 33.925 & 19.374 \\
3 & 0 & 0 \\
4 & 16.222 & 11.519 \\
5 & 23.264 & 4.385 \\
6 & 63.642 & 32.866 \\
7 & 57.116 & 8.033 \\
8 & 5.547 & 4.790 \\
9 & 15.331 & 6.368 \\
10 & $-2.021^{\mathrm{a}}$ & 2.785 \\
11 & 5.129 & 9.759 \\
12 & 15.452 & 4.541 \\
\hline
\end{tabular}

a. A negative value means supplying instead receiving power

There are two other metaheuristic methods used to compare CFA. They are its original FA form and APSO. Table 4 is given to define parameters employed for all used method. In order to define particle's fitness value, here we use weighted sum approach, using cost as the reference.
Since OPF is a constrained problem, here we apply penalties to fitness value for each parameter that not fulfill the constraints given in Section II and III. All simulations are performed by Personal Computer with $3.4 \mathrm{GHz} 4$ core processor and $16 \mathrm{~GB}$ RAM running in Windows 10 operating system. 
Table 4 Parameters used on this paper

\begin{tabular}{cccc}
\hline Parameters & CFA & FA & APSO \\
\hline $\begin{array}{c}\text { Particles/fireflies } \\
\text { used }\end{array}$ & & 20 & \\
Number of & & 20 & \\
Iterations & & $\beta_{0}=1$ & $\alpha=0.5$ \\
& $\beta_{0}=0-2^{\mathrm{a}}$ & $\gamma=0.5$ & $\beta=0.5$ \\
Specialized & $\gamma=0.5$ & $\alpha=0.5-$ & \\
Parameters & $\alpha=0.5-$ & $0.01^{\mathrm{b}}$ & \\
& $0.01^{\mathrm{b}}$ & & \\
\hline
\end{tabular}

a. Changed at the end of iterations. Starting value may be randomized

b. Decreased exponentially

\subsection{Cost minimization}

Our first objective needs to be optimized for reducing generation cost as low as possible. Our simulation result is given in Table 5. All parameters given in Table 5 are rounded at seven digits to help to show their differences.

As can be seen in Table 5, each method gives an extremely close result. It motivates us to look at more numbers behind decimal point at each fitness values. This treatment, along with statistical analysis result, is applied in the next paragraph. However, at least some control variable values are obtained, and their results are almost the same. The result shows the competitiveness of these three methods.

Table 6 shows statistical analysis to compare each method. All numbers given in Table 6 are fourth to ninth number behind decimal point to illustrate their difference more clearly. To help readers to read Table 6, "append" number given there to the right of 1086266.37. For instance, CFA's average fitness value given on Table 6 is 278306. Thus, CFA's average fitness value is 1086266.37. As shown in Table 6, CFA and FA present an extremely close performance, although FA gives more "dense" results. On the other hand, APSO performs a bit worse than two other methods. However, the differences are too small; hence, it may be omitted.

Figure 2 given below is the convergence curve of the best result in this case study. In Figure 2, CFA and FA also have similar convergence characteristics for this study case. This condition is understandable since both CFA and FA share similar movement mechanism. Both of them are converged near the second and third iteration. On the other hand, APSO converged at 16th iteration. Thus, CFA and FA converge better in one iteration than APSO. However, we can't determine that APSO is slower due to here we don't record our simulation time.

\subsection{Power losses reduction}

Another objective considered in this paper is reducing power losses as low as possible. Our simulation result is given in Table 7. All parameters given in Table 7 are rounded at seven digits to assist for showing their differences. Table 7 shows that each method gives an extremely close result once again. In order to clarify the difference between these results, we consider more digits to analyze in the next paragraph. In addition, control variable values are obtained, and they are agreed to each other once more. This value may confirm the competitiveness of these three methods and the flexibility of metaheuristic methods.

Table 5 Best obtained value for cost minimization case study

\begin{tabular}{cccc}
\hline Variables & CFA & FA & APSO \\
\hline$P_{G 3}(\mathrm{MW})$ & 100 & 100 & 100 \\
$P_{G 5}(\mathrm{MW})$ & 80 & 80 & 80 \\
$P_{G 7}(\mathrm{MW})$ & 98.2309653 & 98.2309663 & 98.2309645 \\
$P_{G 12}(\mathrm{MW})$ & 14.9839479 & 14.9839465 & 14.9839482 \\
$Q_{c a p 1}(\mathrm{MVAR})$ & 50 & 50 & 50 \\
Generation Cost & 448085.7735050 & 448085.7731841 & 448085.7737524 \\
Fitness Value & 1086266.3702783 & 1086266.3702783 & 1086266.3702783 \\
\hline
\end{tabular}

Table 6 Statistical analysis result for cost minimization case study

\begin{tabular}{ccccc}
\hline Algorithm & Minimum & Average & Median & Maximum \\
\hline CFA & 278301 & 278306 & 278306 & 278310 \\
FA & 278301 & 278306 & 278306 & 278309 \\
APSO & 278303 & 278309 & 278309 & 278315 \\
\hline
\end{tabular}


Table 7 Best obtained value for loss reduction case study

\begin{tabular}{cccc}
\hline Variables & CFA & FA & APSO \\
\hline$P_{G 3}(\mathrm{MW})$ & 100 & 100 & 100 \\
$P_{G 5}(\mathrm{MW})$ & 80 & 80 & 80 \\
$P_{G 7}(\mathrm{MW})$ & 94.6814344 & 94.6814289 & 94.6814335 \\
$P_{G 12}(\mathrm{MW})$ & 18.4514369 & 18.4514423 & 18.4514378 \\
$Q_{\text {cap } 1}(\mathrm{MVAR})$ & 50 & 50 & 50 \\
Generation Cost & 5.5320096 & 5.5320095 & 5.5320096 \\
Fitness Value & 669492.2024843 & 669492.2024843 & 669492.202484 \\
\hline
\end{tabular}

Table 8 represents the statistical analysis to compare each method for this case study. All numbers given in Table 8 are fourth to the tenth number behind decimal point to illustrate their difference more clearly. To serve readers for reading Table 8, "append" number given there to the right of 669492.202. For instance, CFA's average fitness value given in Table 6 is 4843199. Hence, CFA's average fitness value is 669492.2024843199. In Table 8, CFA gives lower values than other used methods. The result indicates that CFA performs better than FA and APSO for this kind of problem.

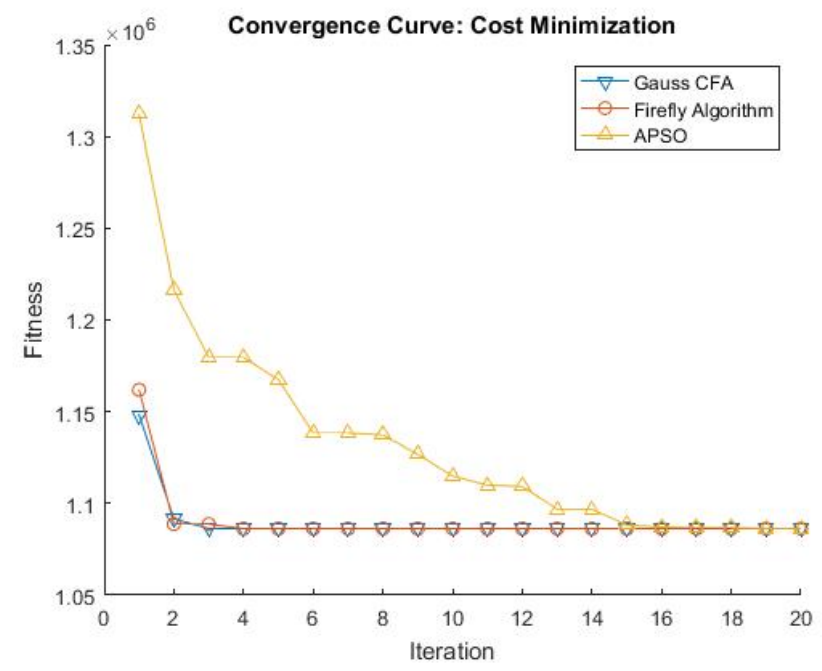

Figure 2 Convergence curve for cost minimization case study

Table 8 Statistical analysis result for loss reduction case study

\begin{tabular}{ccccc}
\hline Algorithm & Minimum & Average & Median & Maximum \\
\hline CFA & 4843156 & 4843199 & 4843199 & 4843228 \\
FA & 4843164 & 4843202 & 4843202 & 4843234 \\
APSO & 4843178 & 4843226 & 4843225 & 4843270 \\
\hline
\end{tabular}

Figure 3 given below, is the convergence curve of the best result in this case study. In Figure 3, CFA and FA also have similar convergence characteristics for this study case once again. This characteristic confirms regarding our statement for similarity on movement mechanism between CFA and FA. Both of them are converged near second iteration.
On the other hand, APSO converged at 15th iteration. It shows that CFA and FA converge better than FA for this study case.

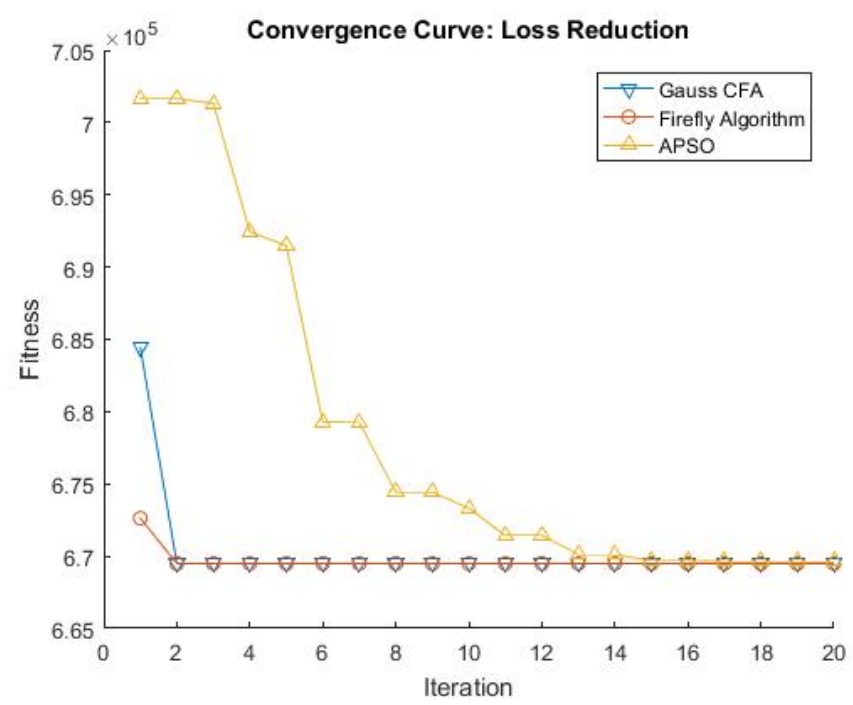

Figure 3 Convergence curve for loss reduction case study

\section{Conclusion}

We successfully implement CFA to solve OPF considering system's voltage stability. For the sake of comparison, we compare this method with the other well-known metaheuristic methods. Our result shows that CFA may perform better than FA to solve OPF problem, while both CFA and FA share similar convergence characteristics. In the future, there would be more advanced methods developed for solving OPF problem and new considerations added that may enrich OPF formulation.

\section{Acknowledgment}

This paper is supported by USAID through Sustainable Higher Education Research Alliances (SHERA) Program - Centre for Collaborative (CCR) National Center for Sustainable Transportation Technology (NCSTT). 


\section{References}

[1] A. J. Wood, B. F. Wollenberg, and G. B. Sheblé, Power Generation, Operation, and Control, Hoboken, New Jersey, John Wiley and Sons, 2014.

[2] F. Milano, Power System Modelling and Scripting, New York, Springer London Dordrecht Heidelberg, 2010.

[3] J. Zhu, Optimization of Power System Operation, 2nd ed., Hoboken, New Jersey, John Wiley \& Sons, 2015.

[4] P. Kundur, Power System Stability and Control, New York, McGraw-Hill, 1994.

[5] T. Ohno, and S. Imai, "The 1987 Tokyo blackout", IEEE PES Power System Conference and Exposition (PSCE), pp. 314-318, 29 October - 1 November 2006.

[6] B. Khan and P. Singh, "Optimal power flow techniques under characterization of conventional and renewable energy sources: a comprehensive analysis", Journal of Engineering, vol. 2017, Article ID 9539506, 16 pages, 2017.

[7] K. Y. Lee, and M. A. El-Sharkawi, Modern Heuristic Optimization Techniques: Theory and Applications to Power Systems, Hoboken, New Jersey, John Wiley \& Sons, 2008.

[8] X. Yang, Engineering Optimization: An Introduction with Metaheuristic Applications, Hoboken, New Jersey, John Wiley \& Sons, 2010.

[9] M. A. Abido, "Optimal power flow using particle swarm optimization", International Journal of Electrical Power \& Energy Systems, vol. 24, no.7, pp. 563-571, 2002.
[10] X. Yang, S. S. S. Hosseini, and A. H. Gandomi, "Firefly Algorithm for solving non-convex economic dispatch problems with valve loading effect", Applied Soft Computing, vol. 12, no. 3, pp. 1880-1186, 2012.

[11] R. Vanitha, J. Baskaran, and M. Sudhakaran, "Multiobjective optimal power flow with STATCOM using DE in WAFGP", Indian Journal of Science and Technology, vol. 8, no. 2, pp. 191-198. 2015.

[12] P. Kessel, and H. Glavitsch, "Estimating the voltage stability of a power system", IEEE Transactions on Power Delivery, vol. 1, no. 3, pp. 346-354. 1986.

[13] H.H. Goh, Q.S. Chua, S.W. Lee, B.C. Kok, K.C. Goh, and K.T.K. Teo, "Evaluation for voltage stability indices in power system using artificial neural network", Procedia Engineering, vol. 118, pp. 1127-1136, 2015.

[14] A. H. Gandomi, X. Yang, S. Talatahari, and A. H. Alavi, "Firefly algorithm with chaos", Communications in Nonlinear Science and Numerical Simulation, vol. 18, no. 1, pp. 89-98, 2013.

[15] X.-S. Yang, "Firefly algorithms for multimodal optimization", Stochastic Algorithms: Foundations and Applications (SAGA), Lecture Notes in Computer Sciences, Vol. 5792, pp. 169-178, 2009.

[16] Y. T. K. Priyanto, and M. Robith, "Economic dispatch and losses minimization using Multi-verse Optimizer on 150 $\mathrm{kV}$ Mahakam transmission system", International Journal of Engineering Research and Technology, vol. 6, no. 1, pp. 489-494, 2017. 